\title{
Model Reduction for Linear Parameter-Dependent Systems
}

\author{
L. $\mathrm{Li}^{*}$ \\ * National ICT Australia, Department of Electrical and Electronic \\ Engineering, The University of Melbourne, VIC, 3010, Australia. \\ (e-mail: li.li@ee.unimelb.edu.au)
}

\begin{abstract}
The paper considers the problem of model reduction for a class of linear parameterdependent (LPD) systems. Three model reduction approaches: balanced truncation, balanced LQG truncation and gain-scheduled $\mathcal{H}_{\infty}$ model reduction, are presented to reduce the dimension of LPD systems. For the former two approaches, conditions to proceed the reduction are given in terms of a finite number of linear matrix inequalities (LMIs); while the latter one involves LMIs with some additional rank constraint.
\end{abstract}

\section{INTRODUCTION}

Model reduction is essential in the analysis and simulation of practical large systems to reduce computation costs. One of the most commonly applied model reduction methods for stable linear time invariant (LTI) systems is the balanced truncation method introduced in Moore (1981); see also Zhou et al. (1996). Based on the controllability and observability grammians for the system under consideration, this method involves finding a state space transformation to simultaneously diagonalize the grammians. This leads to a balanced realization of the system from which the reduced order model is constructed. For unstable LTI systems, a so-called balanced LQG truncation was proposed in Jonckheere and Silverman (1983); see also Mustafa and Glover (1990). This method involves balancing the smallest nonnegative solutions of the standard LQG control and filter algebraic Riccati equations, rather than the two grammians for the balanced truncation method. Being a closed-loop balancing approach, balanced LQG truncation overcomes the restriction on balanced truncation that the original system must be asymptotically stable. Another widely used reduction method is $\mathcal{H}_{\infty}$ model reduction which was first studied in Haddad and Bernstein (1989); Kavranoglu and Bettayeb (1993). In Kavranoglu and Bettayeb (1993), it was shown that generalized controllability and observability grammians can also be used to characterize the $\mathcal{H}_{\infty}$ model reduction problem. $\mathcal{H}_{\infty}$ model reduction was further developed in the framework of linear matrix inequality (LMI), involving some rank constraint; see e.g. Grigoriadis (1995); Skelton et al. (1998); Dullerud and Paganini (2000).

In this paper, we explore the model reduction problems for linear parameter-dependent (LPD) systems, that is, systems affected by time-varying parametric uncertainties. These uncertainties under consideration belong to a polytopic uncertainty set. The results of this paper build on the results in Wang and Balakrishnan (2002) which considers a new unstructured scaling approach to the problem of robust stability and gain-scheduled controller synthesis for LPD systems. A feature of this approach is that it can reduce the conservatism on the stability analysis results, and thus offer significant improvement in practice. This is achieved by employing unstructured scaling matrices at each vertex of the underlying polytopic parameter set, in contrast with conventional techniques which require structured scaling matrices to commute with structured uncertainties (Fan et al., 1991).

We first introduce definitions of controllability and observability grammians of an LPD system. This enables us to develop a balanced truncation model reduction method for the class of LPD systems under consideration. A numerical way to obtain the system grammians is provided in term of a finite number of LMIs, and a balanced truncation algorithm is summarized. A bound on the $\mathcal{H}_{\infty}$ norm error introduced by the model reduction process is also given.

In Prempain (2006), coprime factorizations for LPD systems were considered based on the approach in Wang and Balakrishnan (2002). It is well-known that coprime factorization problems are closely related with LQG control and filter algebraic Riccati equations or inequalities; see e.g. Zhou et al. (1996); Li and Paganini (2005). This fact together with the results of Prempain (2006) motivates the question as to whether a balanced LQG truncation model reduction method can be obtained for LPD systems. In this paper, we derive LMI conditions to obtain the solutions of LQG control and filter algebraic Riccati inequalities for LPD systems. Thus traditional balanced LQG truncation can proceed naturally.

Gain-scheduled $\mathcal{H}_{\infty}$ model reduction for LPD systems is also investigated, based on the techniques used in Wang and Balakrishnan (2002); Apkarian and Gahinet (1995). Different from the conventional gain-scheduled approach in Apkarian and Gahinet (1995), every system matrix of the reduced system is scheduled (Wang and Balakrishnan, 2002). A sufficient condition for the existence of a gainscheduled reduced model is provided which is related to the underlying grammians with some rank constraint.

Notation $M^{T}$ stands for the transpose of a matrix $M$. We use $\operatorname{diag}\left(M_{1}, \cdots, M_{n}\right)$ to denote a block diagonal matrix with matrices $M_{1}, \cdots, M_{n}$ on its main diagonal. 
The state-space realization of a transfer matrix is denoted by $G(s)=\left[\begin{array}{l|l}A & B \\ \hline C & D\end{array}\right]:=C(s I-A)^{-1} B+D$.

\section{PROBLEM FORMULATION}

Let $\boldsymbol{\Theta}$ be a polytope in $\mathbf{R}^{m}$ containing the origin, and consider the uncertainty structure

$$
\begin{gathered}
\boldsymbol{\Delta}=\left\{\operatorname{diag}\left(\theta_{1}(t) I_{s_{1}}, \ldots, \theta_{m}(t) I_{s_{m}}\right):\right. \\
\left.\theta(t)=\left[\theta_{1}(t), \ldots, \theta_{m}(t)\right]^{T} \in \mathbf{\Theta}\right\} .
\end{gathered}
$$

It is obvious that $\boldsymbol{\Delta}$ is a polytope as well, and we denote the vertices of $\boldsymbol{\Delta}$ by $\Delta_{i}, i=1, \cdots, v$.

Consider the following LPD system:

$$
\mathcal{G}_{\theta}:\left\{\begin{array}{l}
\dot{x}=A x+B_{q} q+B_{u} u, \\
p=C_{p} x+D_{p q} q+D_{p u} u, \\
y=C_{y} x+D_{y q} q+D_{y u} u, \\
q=\Delta_{\theta} p, \quad \Delta_{\theta} \in \Delta .
\end{array}\right.
$$

where $x(t) \in \mathbf{R}^{n}$ is the state, $u(t) \in \mathbf{R}^{n_{u}}$ is the control input, $p(t) \in \mathbf{R}^{s}$ is the uncertainty output, $y(t) \in \mathbf{R}^{n_{y}}$ is the measured output and $q(t) \in \mathbf{R}^{s}$ is the uncertainty input; here $s=s_{1}+\cdots+s_{m}$.

Let the nominal system be denoted by

$$
G=\left[\begin{array}{ll}
G_{11} & G_{12} \\
G_{21} & G_{22}
\end{array}\right]=\left[\begin{array}{c|cc}
A & B_{q} & B_{u} \\
\hline C_{p} & D_{p q} & D_{p u} \\
C_{y} & D_{y q} & D_{y u}
\end{array}\right] .
$$

For $\Delta_{u} \in \mathbf{C}^{s}$ such that $I-G_{11} \Delta_{u}$ is non-singular, define

$$
\mathcal{F}_{u}\left(G, \Delta_{u}\right):=G_{22}+G_{21} \Delta_{u}\left(I-G_{11} \Delta_{u}\right)^{-1} G_{12} \text {. }
$$

Similarly, for $\Delta_{l} \in \mathbf{C}^{n_{u} \times n_{y}}$ such that $I-G_{22} \Delta_{l}$ is nonsingular, define

$$
\mathcal{F}_{l}\left(G, \Delta_{l}\right):=G_{11}+G_{12} \Delta_{l}\left(I-G_{22} \Delta_{l}\right)^{-1} G_{21} .
$$

Then, the LPD system (1) is defined by a linear fractional transformation (LFT) as follows,

Here

$$
\mathcal{G}_{\theta}=\mathcal{F}_{u}\left(G, \Delta_{\theta}\right)=\left[\begin{array}{c|c}
\mathcal{A}_{\theta} & \mathcal{B}_{\theta} \\
\hline \mathcal{C}_{\theta} & \mathcal{D}_{\theta}
\end{array}\right], \Delta_{\theta} \in \Delta .
$$

$\left[\begin{array}{ll}\mathcal{A}_{\theta} & \mathcal{B}_{\theta} \\ \mathcal{C}_{\theta} & \mathcal{D}_{\theta}\end{array}\right]=\left[\begin{array}{cc}A & B_{u} \\ C_{y} & D_{y u}\end{array}\right]+\left[\begin{array}{c}B_{q} \\ D_{y q}\end{array}\right] \Delta_{\theta}\left(I-D_{p q} \Delta_{\theta}\right)^{-1}\left[\begin{array}{ll}C_{p} & D_{p u}\end{array}\right]$

The configuration of the LPD system (1) is depicted in Fig. 1.

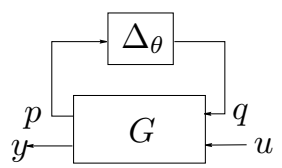

Fig. 1. LFT representation of an uncertain system.

In the following sections we will present three model reduction approaches for the LPD system (1). Before doing that, we recall the definition of quadratic stability, quadratic stabilizability and quadratic detectability for the LPD system (1).

Definition 1. (Wang and Balakrishnan, 2002) An LPD system of the form (1) is said to be quadratically stable if there exists a real positive definite matrix $V=V^{T}>0$ such that

$$
\mathcal{A}_{\theta}^{T} V+V \mathcal{A}_{\theta}<0, \quad \forall \theta \in \boldsymbol{\Theta} .
$$

Alternatively, we say that $\mathcal{A}_{\theta}, \theta \in \boldsymbol{\Theta}$ is quadratically stable.

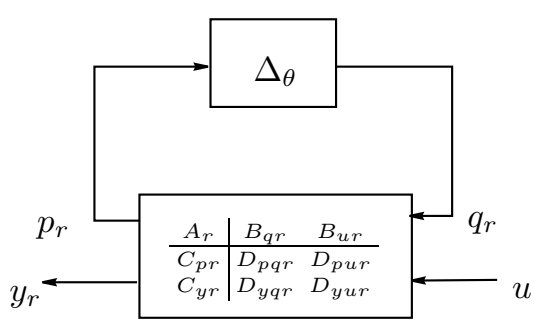

Fig. 2. LFT representation of a reduced uncertain system.

Definition 2. (Prempain, 2006) An LPD system of the form (1) is said to be quadratically stabilizable if there exists a real matrix $F$ such that $\mathcal{A}_{\theta}+\mathcal{B}_{\theta} F$ is quadratically stable. Dually, the system (1) is said to be quadratically detectable if there exists a real matrix $L$ such that $\mathcal{A}_{\theta}+L \mathcal{C}_{\theta}$ is quadratically stable.

\section{BALANCED TRUNCATION}

In this section we present the balanced truncation model reduction method for the LPD system (1). As well known, balanced truncation is one of most popular model reduction methods which is related to the controllability grammian and observability grammian of the underlying system. First we introduce definitions of these grammians for the LPD system (1).

Definition 3. A matrix $W>0$ is said to be a generalized controllability grammian for the LPD system (1) if

$$
\mathcal{A}_{\theta} W+W \mathcal{A}_{\theta}^{T}+\mathcal{B}_{\theta} \mathcal{B}_{\theta}^{T}<0, \quad \forall \theta \in \boldsymbol{\Theta} .
$$

Similarly, a matrix $V>0$ is said to be a generalized observability grammian for the LPD system (1) if

$$
\mathcal{A}_{\theta}^{T} V+V \mathcal{A}_{\theta}+\mathcal{C}_{\theta}^{T} \mathcal{C}_{\theta}<0, \quad \forall \theta \in \boldsymbol{\Theta} .
$$

Here, $\mathcal{A}_{\theta}, \mathcal{B}_{\theta}, \mathcal{C}_{\theta}$ are as defined in (2).

Definition 4. An LPD system of the form (1) is said to be balanced if its generalized observability and controllability grammians are identical diagonal matrices.

Suppose that the LPD system (1) is quadratically stable, as stated in Def. 1. It is straightforward that its generalized controllability and observability grammians exist such that (3) and (4) hold. Then generalized balanced truncation (Dullerud and Paganini, 2000) can be applied to (1) to get reduced model. In what follows sufficient conditions to obtain these grammians for the LPD system (1) are given. Theorem 5. If there exist matrices $P>0, Z>0$ and $M>0$ solving the following semidefinite programming (SDP) problem:

minimize $\operatorname{trace}(Z)$,

$$
\left[\begin{array}{ccc}
(1,1) & P B_{q, i}+C_{p}^{T} M D_{p q, i} & P B_{u}+C_{p}^{T} M D_{p u} \\
\star & -M+D_{p q, i}^{T} M D_{p q, i} & D_{p q, i}^{T} M D_{p u} \\
\star & \star & -I+D_{p u}^{T} M D_{p u}
\end{array}\right]<0,
$$

$$
\left[\begin{array}{ll}
Z & I \\
I & P
\end{array}\right]>0
$$

where $(1,1)=A^{T} P+P A+C_{p}^{T} M C_{p}, B_{q, i}=B_{q} \Delta_{i}$, $D_{p q, i}=D_{p q} \Delta_{i}$, then $P^{-1}$ is a generalized controllability grammian verifying (3). 
Proof. From (1),

$$
\begin{aligned}
& \dot{x}=A x+\left(B_{q} \Delta_{\theta}\right) p+B_{u} u, \\
& p=C_{p} x+\left(D_{p q} \Delta_{\theta}\right) p+D_{p u} u .
\end{aligned}
$$

For any real matrices $G_{\Delta}, H_{\Delta}$ and $K_{\Delta}$ of compatible dimensions, we have

$$
\begin{aligned}
& x^{T} G_{\Delta} p=x^{T} G_{\Delta} C_{p} x+x^{T} G_{\Delta}\left(D_{p q} \Delta_{\theta}\right) p+x^{T} G_{\Delta} D_{p u} u, \\
& p^{T} H_{\Delta} p=p^{T} H_{\Delta} C_{p} x+p^{T} H_{\Delta}\left(D_{p q} \Delta_{\theta}\right) p+p^{T} H_{\Delta} D_{p u} u, \\
& u^{T} K_{\Delta} p=u^{T} K_{\Delta} C_{p} x+u^{T} K_{\Delta}\left(D_{p q} \Delta_{\theta}\right) p+u^{T} K_{\Delta} D_{p u} u .
\end{aligned}
$$

or equivalently

$$
\left[\begin{array}{c}
x \\
p \\
u
\end{array}\right]^{T} \underbrace{\left[\begin{array}{ccc}
G_{\Delta} C_{p}+C_{p}^{T} G_{\Delta}^{T} & \Upsilon_{12} & G_{\Delta} D_{p u}+C_{p}^{T} K_{\Delta}^{T} \\
\star & \Upsilon_{22} & \Upsilon_{23} \\
\star & \star & K_{\Delta} D_{p u}+D_{p u}^{T} K_{\Delta}^{T}
\end{array}\right]}_{\Upsilon}\left[\begin{array}{l}
x \\
p \\
u
\end{array}\right]=0,
$$

where $\Upsilon_{12}=G_{\Delta}\left(D_{p q} \Delta_{\theta}-I\right)+C_{p}^{T} H_{\Delta}^{T}, \Upsilon_{22}=H_{\Delta}\left(D_{p q} \Delta_{\theta}-\right.$ $I)+\left(D_{p q} \Delta_{\theta}-I\right)^{T} H_{\Delta}^{T}$ and $\Upsilon_{23}=H_{\Delta} D_{p u}+\left(D_{p q} \Delta_{\theta}-\right.$ $I)^{T} K_{\Delta}^{T}$. Let $\mathcal{Q}(x, u)=\frac{d}{d t}\left[x^{T}(t) P x(t)\right]-u^{T}(t) u(t)$ which is the quadratic form associated to the $\mathcal{H}_{2}$ problem or equivalently (3). Then,

$$
\begin{aligned}
\mathcal{Q}(x, u) & =\left[\begin{array}{l}
x \\
u
\end{array}\right]^{T}\left[\begin{array}{ccc}
P \mathcal{A}_{\theta}+\mathcal{A}_{\theta}^{T} P & P \mathcal{B}_{\theta} \\
\star & -I
\end{array}\right]\left[\begin{array}{l}
x \\
u
\end{array}\right] \\
& =\left[\begin{array}{l}
x \\
p \\
u
\end{array}\right]^{T} \underbrace{\left[\begin{array}{ccc}
A^{T} P+P A & P\left(B_{q} \Delta_{\theta}\right) & P B_{u} \\
\star & 0 & 0 \\
\star & \star & -I
\end{array}\right]}_{\Gamma}\left[\begin{array}{l}
x \\
p \\
u
\end{array}\right] \\
& =\left[\begin{array}{l}
x \\
p \\
u
\end{array}\right]^{T}(\Gamma+\Upsilon)\left[\begin{array}{l}
x \\
p \\
u
\end{array}\right]
\end{aligned}
$$

Now substitute $G_{\Delta}=C_{p}^{T} M / 2, H_{\Delta}=\left(D_{p q} \Delta_{\theta}+I\right)^{T} M / 2$, $K_{\Delta}=D_{p u}^{T} M / 2$ into $\Upsilon, \mathcal{Q}(x, u)<0$ follows from (5b). This completes the proof.

We also show an alternative proof to see directly that $P^{-1}$ verifies (3).

From (1), we have

$$
\left[\begin{array}{l}
x \\
p \\
u
\end{array}\right]=\left[\begin{array}{cc}
I & 0 \\
C_{p \theta} & D_{p \theta} \\
0 & I
\end{array}\right]\left[\begin{array}{l}
x \\
u
\end{array}\right] \triangleq \Pi\left[\begin{array}{l}
x \\
u
\end{array}\right]
$$

where

$$
\left[\begin{array}{ll}
C_{p \theta} & D_{p \theta}
\end{array}\right]=\left(I-D_{p q} \Delta_{\theta}\right)^{-1}\left[\begin{array}{ll}
C_{p} & D_{p u}
\end{array}\right] .
$$

Following from (5b), $\Gamma+\Upsilon<0$. Note that $\Pi$ hass full column rank, then

$$
\Pi^{T}(\Gamma+\Upsilon) \Pi<0 .
$$

It is easy to check that

$$
\Pi^{T} \Upsilon \Pi=0, \quad \Pi^{T} \Gamma \Pi=\left[\begin{array}{ccc}
P \mathcal{A}_{\theta}+\mathcal{A}_{\theta}^{T} P & P \mathcal{B}_{\theta} \\
\star & & -I
\end{array}\right] .
$$

By Schur complement,

$$
P \mathcal{A}_{\theta}+\mathcal{A}_{\theta}^{T} P+P \mathcal{B}_{\theta} \mathcal{B}_{\theta}^{T} P<0,
$$

therefore, $P^{-1}$ verifies (3).

Theorem 6 . If there exist matrices $\bar{P}>0$ and $\bar{M}>0$ solving the following SDP problem: minimize trace $(\bar{P})$,

$$
\left[\begin{array}{ccc}
\bar{P} A^{T}+A \bar{P}+B_{q, i} \bar{M} B_{q, i}^{T} & \bar{P} C_{p}^{T}+B_{q, i} \bar{M} D_{p q}^{T} & B_{u} \\
\star & -\bar{M}+D_{p q, i} \bar{M} D_{p q, i}^{T} & D_{p u} \\
\star & \star & -I
\end{array}\right]<0,
$$

where $B_{q, i}=B_{q} \Delta_{i}, D_{p q, i}=D_{p q} \Delta_{i}$, then $\bar{P}$ is a generalized controllability grammian verifying (3).

Proof. It suffices to prove that (5b) and (9b) are equivalent with $\bar{P}=P^{-1}$ and $\bar{M}=M^{-1}$.

Rewrite (5b) as

$$
\left[\begin{array}{ccc}
A^{T} P+P A & P B_{q, i} & P B_{u} \\
\star & -M & 0 \\
\star & \star & -I
\end{array}\right]+\left[\begin{array}{c}
C_{p}^{T} \\
D_{p q, i}^{T} \\
D_{p u}^{T}
\end{array}\right] M\left[\begin{array}{c}
C_{p}^{T} \\
D_{p q, i}^{T} \\
D_{p u}^{T}
\end{array}\right]^{T}<0
$$

which is equivalent to

$$
\left[\begin{array}{cccc}
A^{T} P+P A & P B_{q, i} & P B_{u} & C_{p}^{T} \\
\star & -M & 0 & D_{p q, i}^{T} \\
\star & \star & -I & D_{p u}^{T} \\
\star & \star & \star & -M^{-1}
\end{array}\right]<0
$$

Left and right multiply (10) with $\operatorname{diag}\left(P^{-1}, I, I, I\right)$ and let $\bar{P}=P^{-1}$ and $\bar{M}=M^{-1}$, then

$$
\left[\begin{array}{cccc}
\bar{P} A^{T}+A \bar{P} & B_{q, i} & B_{u} & \bar{P} C_{p}^{T} \\
\star & -\bar{M}^{-1} & 0 & D_{p q, i}^{T} \\
\star & \star & -I & D_{p u}^{T} \\
\star & \star & \star & -\bar{M}
\end{array}\right]<0
$$

Apply Schur complement to (11) with respect to its $(2,2)$ block to obtain (9b).

To obtain a generalized observability grammian, we apply Theorem 5 and 6 to the dual system of (1).

Theorem \%. If there exist matrices $Q>0, Z>0$ and $N>0$ solving the following SDP problem:

minimize $\operatorname{trace}(Z)$,

$$
\left[\begin{array}{ccc}
(1,1) & Q \tilde{C}_{p, i}^{T}+B_{q} N \tilde{D}_{p q, i}^{T} & Q C_{y}^{T}+B_{q} N D_{y q}^{T} \\
\star & -N+\tilde{D}_{p q, i} N \tilde{D}_{p q, i}^{T} & D_{p q, i} N D_{y q}^{T} \\
\star & \star & -I+D_{y q} N D_{y q}^{T}
\end{array}\right]<0,
$$

$$
\left[\begin{array}{ll}
Z & I \\
I & Q
\end{array}\right]>0
$$

where $(1,1)=A Q+Q A^{T}+B_{q} N B_{q}^{T}, \tilde{C}_{p, i}=\Delta_{i} C_{p}$, $\tilde{D}_{p q, i}=\Delta_{i} D_{p q}$, then $Q^{-1}$ is a generalized observability grammian verifying (4).

Theorem 8. If there exist matrices $\bar{Q}>0$ and $\bar{N}>0$ solving the following SDP problem: minimize $\operatorname{trace}(\bar{Q})$,

$$
\begin{array}{ccc}
{\left[\begin{array}{ccc}
(1,1) & \bar{Q} B_{q}+\tilde{C}_{p, i}^{T} \bar{N} \tilde{D}_{p q, i} & C_{y}^{T} \\
\star & -\bar{N}+\tilde{D}_{p q, i}^{T} \bar{N} \tilde{D}_{p q, i} & D_{y q}^{T} \\
\star & \star & -I
\end{array}\right]<0,} \\
\quad i=1, \ldots, v,
\end{array}
$$

where $(1,1)=\bar{Q} A+A^{T} \bar{Q}+\tilde{C}_{p, i}^{T} \bar{N} \tilde{C}_{p, i}, \tilde{C}_{p, i}=\Delta_{i} C_{p}$, $\tilde{D}_{p q, i}=\Delta_{i} D_{p q}$, then $\bar{Q}$ is a generalized observability grammian verifying (4).

We summarize the proposed balanced truncation model reduction algorithm as follows. 
Procedure 9. (Balanced Truncation).

(1) Solve SDP problems (9) and (13) to obtain generalized grammians $\bar{P}, \bar{Q}$.

(2) Balance $\bar{P}, \bar{Q}$ by a transformation matrix $T$ such that $T \bar{P} T^{T}=\left(T^{-1}\right)^{T} \bar{Q} T^{-1}=\Sigma=\operatorname{diag}\left(\Sigma_{1}, \Sigma_{2}\right)=$ $\operatorname{diag}\left(\gamma_{1}, \ldots, \gamma_{n}\right)$, where $\gamma_{1} \geq \ldots \geq \gamma_{k}>\gamma_{k+1} \geq \ldots \geq$ $\gamma_{n}>0, \Sigma_{1}=\operatorname{diag}\left(\gamma_{1}, \ldots, \gamma_{k}\right), \Sigma_{2}=\operatorname{diag}\left(\gamma_{k+1}, \ldots, \gamma_{n}\right)$.

(3) Write the transformed nominal system of (1) as

$$
G=\left[\begin{array}{c|cc}
\bar{A} & \bar{B}_{q} & \bar{B}_{u} \\
\hline C_{p} & D_{p q} & D_{p u} \\
\bar{C}_{y} & D_{y q} & D_{y u}
\end{array}\right]
$$

where

$$
\begin{aligned}
\bar{A} & =T A T^{-1} ; \quad \bar{B}_{q}=T B_{q} ; \quad \bar{B}_{u}=T B_{u} \\
\bar{C}_{p} & =C_{p} T^{-1} ; \quad \bar{C}_{y}=C_{y} T^{-1} .
\end{aligned}
$$

The sub-matrices of $G$ corresponding to $\Sigma_{2}$ are truncated to obtain the reduced system

$$
G_{r}=\left[\begin{array}{c|cc}
\bar{A}_{r} & \bar{B}_{q r} & \bar{B}_{u r} \\
\hline C_{p r} & D_{p q} & D_{p u} \\
\bar{C}_{y r} & D_{y q} & D_{y u}
\end{array}\right]
$$

with order $k$.

(4) Write the reduced system of (1) as $\mathcal{G}_{r \theta}=\mathcal{F}_{u}\left(G_{r}, \Delta_{\theta}\right)$, $\Delta_{\theta} \in \Delta$.

Theorem 10. Suppose that $\mathcal{G}_{r \theta}$ is obtained as described in Procedure 9 , then $\mathcal{G}_{r \theta}$ is quadratically stable. Furthermore,

$$
\left\|\mathcal{G}_{\theta}(s)-\mathcal{G}_{r \theta}(s)\right\|_{\infty} \leq 2\left(\gamma_{1}^{t}+\cdots+\gamma_{l}^{t}\right),
$$

where $\gamma_{i}^{t}$ denote the distinct generalized Hankel singular values of $\gamma_{k+1}, \ldots, \gamma_{n}$, that is, $\gamma_{1}^{t}>\gamma_{2}^{t}>\ldots>\gamma_{l}^{t}$ and $\left\{\gamma_{k+1}, \ldots, \gamma_{n}\right\}=\left\{\gamma_{1}^{t}, \ldots, \gamma_{l}^{t}\right\}$.

Proof. Partition $G$ in (14) accordingly as

$$
G=\left[\begin{array}{cc|cc}
\bar{A}_{r} & \bar{A}_{12} & \bar{B}_{q r} & \bar{B}_{u r} \\
\bar{A}_{21} & \bar{A}_{22} & \bar{B}_{q 2} & \bar{B}_{u 2} \\
\hline C_{p r} & C_{p 2} & D_{p q} & D_{p u} \\
\bar{C}_{y r} & \bar{C}_{y 2} & D_{y q} & D_{y u}
\end{array}\right] .
$$

Then it follows from Theorems 6 and 8 that $\Sigma=$ $\operatorname{diag}\left(\Sigma_{1}, \Sigma_{2}\right)$ is the balanced grammian of the LPD system $\mathcal{F}_{u}\left(G, \Delta_{\theta}\right)=$

$$
\left[\begin{array}{cc|c}
\bar{A}_{r}+\bar{B}_{q r} \hat{\Delta}_{\theta} \bar{C}_{p r} & \bar{A}_{12}+\bar{B}_{q r} \hat{\Delta}_{\theta} \bar{C}_{p 2} & \bar{B}_{u r}+\bar{B}_{q r} \hat{\Delta}_{\theta} D_{p u} \\
\bar{A}_{21}+\bar{B}_{q 2} \hat{\Delta}_{\theta} \bar{C}_{p r} & \bar{A}_{22}+\bar{B}_{q 2} \hat{\Delta}_{\theta} \bar{C}_{p 2} & \bar{B}_{u 2}+\bar{B}_{q 2} \hat{\Delta}_{\theta} D_{p u} \\
\hline \bar{C}_{y r}+D_{y q} \hat{\Delta}_{\theta} \bar{C}_{p r} & \bar{C}_{y 2}+D_{y q} \hat{\Delta}_{\theta} \bar{C}_{p 2} & D_{y u}+D_{y q} \hat{\Delta}_{\theta} D_{p u}
\end{array}\right],
$$
where $\hat{\Delta}_{\theta}=\Delta_{\theta}\left(I-D_{p q} \Delta_{\theta}\right)^{-1}$.

The reduced dimension uncertain system $\mathcal{G}_{r \theta}$ corresponding to $\Sigma_{1}$ is

$$
\mathcal{F}_{u}\left(G_{r}, \Delta_{\theta}\right)=\left[\begin{array}{c|c}
\bar{A}_{r}+\bar{B}_{q r} \hat{\Delta}_{\theta} \bar{C}_{p r} & \bar{B}_{u r}+\bar{B}_{q r} \hat{\Delta}_{\theta} D_{p u} \\
\hline \bar{C}_{y r}+D_{y q} \hat{\Delta}_{\theta} \bar{C}_{p r} & D_{y u}+D_{y q} \hat{\Delta}_{\theta} D_{p u}
\end{array}\right],
$$

where $G_{r}$ is defined in (15).

It is easy to show that $\mathcal{G}_{r \theta}$ satisfies (3) and (4) with balanced grammian $\Sigma_{1}$. Therefore, $\mathcal{G}_{r \theta}$ is balanced and quadratically stable. The bound in (16) holds from the traditional balanced truncation technique, see e.g. Dullerud and Paganini (2000).

\section{BALANCED LQG TRUNCATION}

The balanced truncation technique, as introduced in the last section, requires the LPD system to be quadratically stable. This restriction may be due to the open-loop balancing (Jonckheere and Silverman, 1983). A new balanced LQG truncation approach, taking into account of the closed-loop control considerations, was presented in Jonckheere and Silverman (1983) to overcome the restriction on unstable LTI systems. The approach involves balancing the smallest nonnegative solutions of the standard LQG control and filter Riccati equations. The readers are referred to Jonckheere and Silverman (1983) for more details and discussions. In this section, we apply this approach to the LPD system (1) which may be quadratically unstable.

Suppose that the LPD system (1) is quadratically stabilizable and quadratically detectable, as stated in Def. 2. Consider the following LQG control and filter Riccati inequalities for the LPD system (1),

$$
\begin{array}{r}
W\left(\mathcal{A}_{\theta}-\mathcal{B}_{\theta} \mathcal{R}_{\theta}^{-1} \mathcal{D}_{\theta}^{T} \mathcal{C}_{\theta}\right)+\left(\mathcal{A}_{\theta}-\mathcal{B}_{\theta} \mathcal{R}_{\theta}^{-1} \mathcal{D}_{\theta}^{T} \mathcal{C}_{\theta}\right)^{T} W \\
-W \mathcal{B}_{\theta} \mathcal{R}_{\theta}^{-1} \mathcal{B}_{\theta}^{T} W+\mathcal{C}_{\theta}^{T} \tilde{\mathcal{R}}_{\theta}^{-1} \mathcal{C}_{\theta}<0, \forall \theta \in \boldsymbol{\Theta}, \\
\left(\mathcal{A}_{\theta}-\mathcal{B}_{\theta} \mathcal{R}_{\theta}^{-1} \mathcal{D}_{\theta}^{T} \mathcal{C}_{\theta}\right) V+V\left(\mathcal{A}_{\theta}-\mathcal{B}_{\theta} \mathcal{R}_{\theta}^{-1} \mathcal{D}_{\theta}^{T} \mathcal{C}_{\theta}\right)^{T} \\
-V \mathcal{C}_{\theta}^{T} \tilde{\mathcal{R}}_{\theta}^{-1} \mathcal{C}_{\theta} V+\mathcal{B}_{\theta} \mathcal{R}_{\theta}^{-1} \mathcal{B}_{\theta}^{T}<0, \forall \theta \in \boldsymbol{\Theta},
\end{array}
$$

where $\mathcal{R}_{\theta}=I+\mathcal{D}_{\theta}^{T} \mathcal{D}_{\theta}, \tilde{\mathcal{R}}_{\theta}=I+\mathcal{D}_{\theta} \mathcal{D}_{\theta}^{T}$.

Definition 11. An LPD system of the form (1) is said to be $L Q G$-balanced if there exist matrices $W>0, V>$ 0 , satisfying (17) and (18) such that they are identical diagonal matrices.

For an LTI system, it is shown that LQG control and filter algebraic Riccati equations or inequalities are closely related to coprime factorization problems (Zhou et al., 1996; Li and Paganini, 2005) and some special $\mathcal{H}_{2}$ control problems (Doyle et al., 1989). In Prempain (2006), coprime factorizations for LPD systems were discussed, and the problems were also reduced to some $\mathcal{H}_{2}$ problems for LPD systems. All these facts motivates the question as to whether the LQG control and filter Riccati inequalities (17) and (18) for LPD systems can be solved by the same $\mathcal{H}_{2}$ problems in Prempain (2006). In what follows, we will show such relationships and provide a numerical approach to obtain the solution to Riccati inequalities (17) and (18).

Motivated by Prempain (2006); Doyle et al. (1989), the filter Riccati inequality (18) is related to the output injection $\mathcal{H}_{2}$ problem, that is, finding a static output feedback gain $L$, such that $\left\|\mathcal{F}_{l}\left(\mathcal{G}_{O I \theta}, L\right)\right\|_{\mathcal{H}_{2}}$ is minimized, where

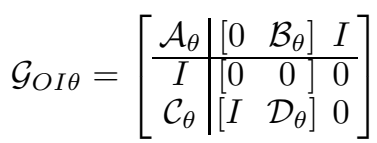

with the following realization,

$$
\mathcal{G}_{O I \theta}:\left\{\begin{array}{l}
\dot{x}=A x+B_{q} q+B_{u} u_{2}+w, \\
p=C_{p} x+D_{p q} q+D_{p u} u_{2}, \\
y=x, \\
z=C_{y} x+D_{y q} q+u_{1}+D_{y u} u_{2}, \\
q=\Delta_{\theta} p, \quad \Delta_{\theta} \in \Delta .
\end{array}\right.
$$

We can now apply Theorem 5 to (19) with $w=L z$, that is, substitute the following parameters into (5),

$$
A+L C_{y} \rightarrow A, B_{q}+L D_{y q} \rightarrow B_{q},
$$$$
\left[\begin{array}{ll}
L & B_{u}+L D_{y u}
\end{array}\right] \rightarrow B_{u},\left[\begin{array}{ll}
0 & D_{p u}
\end{array}\right] \rightarrow D_{p u} .
$$

Letting $Y=P L$, then we have the following result. 
Theorem 12. If there exist matrices $P>0, Z>0, M>0$ and $Y$ solving the following SDP problem:

minimize $\operatorname{trace}(Z)$,

$$
\left[\begin{array}{cccc}
(1,1) & (1,2) & Y & P B_{u}+Y D_{y u}+C_{p}^{T} M D_{p u} \\
\star & (2,2) & 0 & D_{p q, i}^{T} M D_{p u} \\
\star & \star & -I & 0 \\
\star & \star & \star & -I+D_{p u}^{T} M D_{p u}
\end{array}\right]<0
$$$$
\left[\begin{array}{ll}
Z & I \\
I & P
\end{array}\right]>0
$$

where $(1,1)=A^{T} P+P A+Y C_{y}+C_{y}^{T} Y^{T}+C_{p}^{T} M C_{p}$ $(1,2)=P B_{q, i}+Y D_{y q, i}+C_{p}^{T} M D_{p q, i},(2,2)=-M+$ $D_{p q, i}^{T} M D_{p q, i}, B_{q, i}=B_{q} \Delta_{i}, D_{y q, i}=D_{y q} \Delta_{i}, D_{p q, i}=$ $D_{p q} \Delta_{i}$, then $P^{-1}$ verifies (18).

Proof. Following the same routine of the proof of Theorem 5 with substitution (20), we use the following $\Pi$

$$
\Pi=\left[\begin{array}{ccc}
I & 0 & 0 \\
C_{p \theta} & 0 & D_{p \theta} \\
0 & I & 0 \\
0 & 0 & I
\end{array}\right]
$$

where $C_{p \theta}, D_{p \theta}$ is defined in (7).

From (8), with substitution (20) and $L=P^{-1} Y$, we have

$$
\begin{aligned}
P\left(\mathcal{A}_{\theta}+L \mathcal{C}_{\theta}\right) & +\left(\mathcal{A}_{\theta}+L \mathcal{C}_{\theta}\right)^{T} P \\
& +P\left[\begin{array}{ll}
L & \mathcal{B}_{\theta}+L \mathcal{D}_{\theta}
\end{array}\right]\left[\begin{array}{ll}
L & \mathcal{B}_{\theta}+L \mathcal{D}_{\theta}
\end{array}\right]^{T} P<0
\end{aligned}
$$

which is

$$
\begin{aligned}
& P\left(\mathcal{A}_{\theta}-\mathcal{B}_{\theta} \mathcal{R}_{\theta}^{-1} \mathcal{D}_{\theta}^{T} \mathcal{C}_{\theta}\right)+\left(\mathcal{A}_{\theta}-\mathcal{B}_{\theta} \mathcal{R}_{\theta}^{-1} \mathcal{D}_{\theta}^{T} \mathcal{C}_{\theta}\right)^{T} P \\
& -\mathcal{C}_{\theta}^{T} \tilde{\mathcal{R}}_{\theta}^{-1} \mathcal{C}_{\theta}+P \mathcal{B}_{\theta} \mathcal{R}_{\theta}^{-1} \mathcal{B}_{\theta}^{T} P \\
& +\left(\tilde{\mathcal{R}}_{\theta} Y+\mathcal{C}_{\theta}+\mathcal{D}_{\theta} \mathcal{B}_{\theta}^{T} P\right)^{T} \tilde{\mathcal{R}}_{\theta}^{-1}\left(\tilde{\mathcal{R}}_{\theta} Y+\mathcal{C}_{\theta}+\mathcal{D}_{\theta} \mathcal{B}_{\theta}^{T} P\right)<0 .
\end{aligned}
$$

This implies that $P^{-1}$ verifies (18).

Similarly, a solution to the control Riccati inequality (17) is related to the following result.

Theorem 13. If there exist matrices $Q>0, Z>0, N>0$ and $X$ solving the following SDP problem:

minimize $\operatorname{trace}(Z)$,

$$
\begin{aligned}
& {\left[\begin{array}{cccc}
(1,1) & (1,2) & X^{T} & Q C_{y}^{T}+X^{T} D_{y u}^{T}+B_{q} N D_{y q}^{T} \\
\star & (2,2) & 0 & \tilde{D}_{p q, i} N D_{y q}^{T} \\
\star & \star & -I & 0 \\
\star & \star & \star & -I+D_{y q} N D_{y q}^{T}
\end{array}\right]<0} \\
& {\left[\begin{array}{cc}
Z & I \\
I & Q
\end{array}\right]>0,}
\end{aligned}
$$

where $(1,1)=A Q+Q A^{T}+B_{u} X+X^{T} B_{u}^{T}+B_{q} N B_{q}^{T}$, $(1,2)=Q \tilde{C}_{p, i}^{T}+X^{T} \tilde{D}_{p u, i}^{T}+B_{q} N \tilde{D}_{p q, i}^{T},(2,2)=-N+$ $\tilde{D}_{p q, i} N \tilde{D}_{p q, i}^{T}, \quad \tilde{C}_{p, i}=\Delta_{i} C_{p}, \tilde{D}_{p u, i}=\Delta_{i} D_{p u}, \tilde{D}_{p q, i}=$ $\Delta_{i} D_{p q}$, then $Q^{-1}$ verifies (17).

We now summarize the proposed balanced LQG truncation algorithm as follows.

Procedure 14. (Balanced LQG Truncation).

(1) Solve SDP problems (21) and (22) to obtain $P$ and $Q$, and let $\bar{P}=P^{-1}, \bar{Q}=Q^{-1}$

(2) Follow Steps 2-4 in Procedure 9.

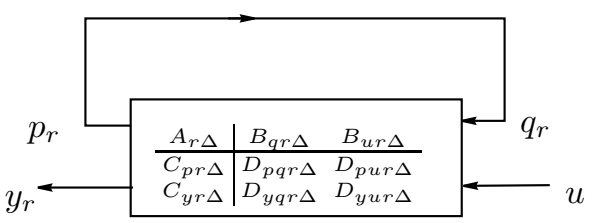

Fig. 3. LFT representation of a gain-scheduled reduced uncertain system.

\section{GAIN-SCHEDULED $\mathcal{H}_{\infty}$ MODEL REDUCTION}

As seen in the last two sections, two balanced truncation approaches to model reduction for LPD systems are discussed. In this section we consider the $\mathcal{H}_{\infty}$ model reduction problem for an LPD system $\mathcal{G}_{\theta}$ of the form (1). Namely, we seek a reduced order system $\mathcal{G}_{r \theta}$ such that $\sup \| \mathcal{G}_{\theta}-$ $\Delta_{\theta} \in \Delta$ $\mathcal{G}_{r \theta} \|_{\infty} \leq \gamma$, where $\gamma$ is a preselected positive number.

To this end, we first state a version of bounded real lemma for LPD systems as follows.

Theorem 15. Given $\mathcal{G}_{\theta}(1)$, if there exist matrices $P>0$, $Z>0$ and $M>0$ satisfying

$$
\begin{aligned}
& {\left[\begin{array}{ccc}
(1,1)(1,2) & P B_{u}+C_{y}^{T} D_{y u}+C_{p}^{T} M D_{p u} \\
\star & (2,2) & D_{y q, i}^{T} D_{y u}+D_{p q, i}^{T} M D_{p u} \\
\star & \star & -\gamma^{2} I+D_{y u}^{T} D_{y u}+D_{p u}^{T} M D_{p u}
\end{array}\right]<0} \\
& i=1, \ldots, v,
\end{aligned}
$$

where $(1,1)=A^{T} P+P A+C_{y}^{T} C_{y}+C_{p}^{T} M C_{p},(1,2)=$ $P B_{q, i}+C_{y}^{T} D_{y q, i}+C_{p}^{T} M D_{p q, i},(2,2)=-M+D_{y q, i}^{T} D_{y q, i}+$ $D_{p q, i}^{T} M D_{p q, i}, B_{q, i}=B_{q} \Delta_{i}, D_{y q, i}=D_{y q} \Delta_{i}, D_{p q, i}=$ $D_{p q} \Delta_{i}$, then $\sup _{\Delta_{\theta} \in \Delta}\left\|\mathcal{G}_{\theta}\right\|_{\infty} \leq \gamma$.

Proof. Similar to the proof of Theorem 5, from (23), it is easy to verify

$$
\left[\begin{array}{cc}
P \mathcal{A}_{\theta}+\mathcal{A}_{\theta}^{T} P+\mathcal{C}_{\theta}^{T} \mathcal{C}_{\theta} & P \mathcal{B}_{\theta}+\mathcal{C}_{\theta}^{T} \mathcal{D}_{\theta} \\
\star & -\gamma^{2} I+\mathcal{D}_{\theta}^{T} \mathcal{D}_{\theta}
\end{array}\right]<0 .
$$

Therefore, by the traditional bounded real lemma, $\left\|\mathcal{G}_{\theta}\right\|_{\infty} \leq$ $\gamma$ for every $\Delta_{\theta} \in \Delta$.

The gain-scheduled reduced system under consideration, as shown in Fig. 3, has the following realization:

$$
\mathcal{G}_{r \theta}:\left\{\begin{array}{l}
\dot{x}_{r}=A_{r \Delta} x_{r}+B_{q r \Delta} q_{r}+B_{u r \Delta} u, \\
p_{r}=C_{p r \Delta} x+D_{p q r} q_{r}+D_{p u r \Delta} u, \\
y_{r}=C_{y r \Delta} x+D_{y q r \Delta} q_{r}+D_{y u r \Delta} u, \\
q_{r}=p_{r} .
\end{array}\right.
$$

Here the subscript $\Delta$ denotes that the system matrices are functions of the time-varying matrix $\Delta_{\theta}$. Different from the one in Fig. 2, every system matrix in (24) is scheduled. The scheme is originally presented in Wang and Balakrishnan (2002), which incorporates uncertainties into system matrices, but still keep the unity feedback. In such a way, the reduced system inherits a similar structure from the original system, and consequently makes it possible to apply Theorem 15. Moreover, this unity feedback is essential as it enables us to establish some LMI-like conditions for the existence of the gain-scheduled reduced system.

Now we are in the position to present a condition to $\mathcal{H}_{\infty}$ model reduction for LPD systems. 
Theorem 16. Given a quadratically stable LPD system $\mathcal{G}_{\theta}$ $(1)$, there exists a reduced LPD system $\mathcal{G}_{r \theta}(24)$ of order $d$ such that

$$
\sup _{\Delta_{\theta} \in \Delta}\left\|\mathcal{G}_{\theta}-\mathcal{G}_{r \theta}\right\|_{\infty} \leq \gamma,
$$

if there exist $\hat{P}>0, \hat{Q}>0, \hat{M}>0, \hat{N}>0$ solving the following rank constrained LMIs

$$
\begin{aligned}
& {\left[\begin{array}{ccc}
\mathcal{M}_{11} & \hat{P} C_{p}^{T}+B_{q, i} \hat{M} D_{p q, i}^{T} & B_{u} \\
\star & -\hat{M}+D_{p q, i} \hat{M} D_{p q, i}^{T} & D_{p u} \\
\star & \star & -\gamma^{2} I
\end{array}\right]<0, } \\
& \star {\left[\begin{array}{ccc}
\mathcal{N}_{11} & \hat{Q} B_{q, i}+C_{p}^{T} \hat{N} D_{p q, i} & C_{y}^{T} \\
\star & -\hat{N}+D_{p q, i}^{T} \hat{N} D_{p q, i} & D_{y q, i}^{T} \\
\star & \star & -I
\end{array}\right]<0, } \\
& {\left[\begin{array}{cc}
\hat{M} & I \\
I & \hat{N}
\end{array}\right] \geq 0, \quad\left[\begin{array}{ll}
\hat{P} & I \\
I & \hat{Q}
\end{array}\right] \geq 0, \quad \operatorname{rank}\left[\begin{array}{cc}
\hat{P} & I \\
I & \hat{Q}
\end{array}\right] \leq n+d, } \\
& i=1, . ., v,
\end{aligned}
$$

where $\mathcal{M}_{11}=\hat{P} A^{T}+A \hat{P}+B_{q, i} \hat{M} B_{q, i}^{T}, \mathcal{N}_{11}=\hat{Q} A+A^{T} \hat{Q}+$ $C_{p}^{T} \hat{N} C_{p}, B_{q, i}=B_{q} \Delta_{i}, D_{p q, i}=D_{p q} \Delta_{i}, D_{y q, i}=D_{y q} \Delta_{i}$.

The proof and the construction of the gain-scheduled reduced system is a routine procedure, which uses the Elimination Lemma and the Completion Lemma, and thus omitted here. The readers are referred to Wang and Balakrishnan (2002) and Apkarian and Gahinet (1995) for details.

Remark 17. Note that (25) is equivalent to (9b) by letting $\hat{P}=\gamma^{-2} \bar{P}$ and $\hat{M}=\gamma^{-2} \bar{M}$. Thus $\gamma^{2} \hat{P}$ is a generalized controllability grammian of the LPD system $\mathcal{G}_{\theta}$ (1). Also, (26) is of the similar form to (13b); the only difference is that $G \circ \Delta_{\theta}$ is used in (26) while $\Delta_{\theta} \circ G$ used in (13b). It is easy to verify that $\hat{Q}$ is also a generalized observability grammian satisfying (4) by following an argument similar to the proof of Theorem 5. Therefore, Theorem 16 states a sufficient condition actually involving two system grammians and some rank condition. This is parallel to the results for a nominal system without uncertainties as shown in (Dullerud and Paganini, 2000, Theorem 4.20), that is, generalized grammians can also be used to characterize the $\mathcal{H}_{\infty}$ model reduction problem; see also the original paper Kavranoglu and Bettayeb (1993). Similar result can also be found in Li and Petersen (2007).

\section{CONCLUSION}

We have proposed a series of model reduction methods for LPD systems. Firstly, definitions of controllability and observability grammians of an LPD system are introduced, and LMI conditions to obtain these grammians are provided. This allows developing a balanced truncation model reduction method for the class of LPD systems under consideration. A bound on the $\mathcal{H}_{\infty}$ norm error introduced by the model reduction process is also given. Secondly, based on the relationships between control and filter algebraic Riccati equations or inequalities and some special $\mathcal{H}_{2}$ control problems, a numerical approach to obtain the solution to Riccati inequalities is derived. Thus traditional balanced LQG truncation can proceed naturally. Thirdly, gain-scheduled $\mathcal{H}_{\infty}$ model reduction for LPD systems is investigated. A sufficient condition for the existence of a gain-scheduled reduced model is provided which is parallel to the results for a nominal system without uncertainties. This condition is related to the underlying grammians with some rank constraint.

\section{REFERENCES}

P. Apkarian and P. Gahinet. A convex characterization of gain-scheduled $H_{\infty}$ controllers. IEEE Transactions on Automatic Control, 40(5):853-864, 1995.

J. C. Doyle, K. Glover, P. P. Khargonekar, and B. A. Francis. State-space solutions to standard $H_{2}$ and $H_{\infty}$ control problems. IEEE Transactions on Automatic Control, 34(8):831-847, 1989.

G. E. Dullerud and F. Paganini. A Course in Robust Control Theory : a Convex Approach. Springer, New York, 2000.

M. K. H. Fan, A. L. Tits, and J. C. Doyle. Robustness in the presence of mixed parametric uncertainty and unmodeled dynamics. IEEE Transactions on Automatic Control, 36(1):25-38, 1991.

K. M. Grigoriadis. Optimal $H_{\infty}$ model reduction via linear matrix inequalities: continuous- and discrete-time cases. Systems \& Control Letters, 26(5):321-333, 1995.

W. M. Haddad and D. S. Bernstein. Combined $L_{2} / H_{\infty}$ model reduction. International Journal of Control, 49 (5):1523-1535, 1989.

E. Jonckheere and L. Silverman. A new set of invariants for linear systems-application to reduced order compensator design. IEEE Transactions on Automatic Control, 28(10):953-964, 1983.

D. Kavranoglu and M. Bettayeb. Characterization of the solution to the optimal $H_{\infty}$ model reduction problem. Systems \& Control Letters, 20(2):99-107, 1993.

L. Li and F. Paganini. Structured coprime factor model reduction based on LMIs. Automatica, 41(1):145-151, 2005.

L. Li and I. R. Petersen. A gramian-based approach to model reduction for uncertain systems. In 46th IEEE Conference on Decision and Control, to appear, 2007.

B.C. Moore. Principal component analysis in linear systems: controllability, observablity, and model reduction. IEEE Transactions on Automatic Control, 26:17-32, 1981.

D. Mustafa and K. Glover. Minimum Entropy $H_{\infty}$ Control. Springer-Verlag, Berlin, 1990.

E. Prempain. On coprime factors for parameter-dependent systems. In 45th IEEE Conference on Decision and Control, pages 5796-5800, 2006.

R. E. Skelton, T. Iwasaki, and K. M. Grigoriadis. A unified algebraic approach to linear control design. Taylor \& Francis Ltd., London, 1998.

F. Wang and V. Balakrishnan. Improved stability analysis and gain-scheduled controller synthesis for parameterdependent systems. IEEE Transactions on Automatic Control, 47(5):720-734, 2002.

K. Zhou, J. Doyle, and K. Glover. Robust and Optimal Control. Prentice-Hall, Upper Saddle River, NJ, 1996. 